\title{
Adaptação transcultural e análise das propriedades psicométricas da versão brasileira do instrumento Motor Activity Log
}

\author{
Viviane Amaral Saliba, ${ }^{1}$ Lívia de Castro Magalhães, ${ }^{2}$ \\ Christina Danielli Coelho de Morais Faria, ${ }^{3}$ \\ Glória Elizabeth Carneiro Laurentino, ${ }^{4}$ Janine Gomes Cassiano ${ }^{2}$ \\ e Luci Fuscaldi Teixeira-Salmela ${ }^{3}$
}

Como citar Saliba VA, Magalhães LC, Faria CDCM, Laurentino GEC, Cassiano JG, Teixeira-Salmela LF. Adaptação transcultural e análise das propriedades psicométricas da versão brasileira do instrumento Motor Activity Log. Rev Panam Salud Publica. 2011:30(3):262-71.

RESUMO Objetivo. Traduzir para o português brasileiro e adaptar transculturalmente o Motor Activity $\log (M A L)$, instrumento específico que avalia a função do membro superior mais afetado (MSMA) em hemiplégicos.

Métodos. O MAL foi traduzido e adaptado seguindo instruções padronizadas e submetido a exame de confiabilidade teste-reteste (coeficiente de correlação intraclasse, CCI). As demais propriedades psicométricas foram investigadas pela análise de Rasch em 77 hemiplégicos crônicos (41 homens, média de idade $=57,5 \pm 12,4$ anos).

Resultados. Tanto a escala de quantidade como a escala de qualidade do MAL obtiveram excelente CCI $(0,98)$ para as pontuações totais. Quanto ao grau de dificuldade, "usar a chave para destrancar a porta" foi o item mais difícil na escala quantitativa, sendo "lavar as mãos" o item mais fácil. Na escala qualitativa, o mais difícil foi "utilizar o controle remoto da TV", $e$ o mais fácil, "secar as mãos". A análise mostrou que o conjunto dos itens enquadrou-se no modelo; entretanto, quatro itens não se encaixaram nas expectativas do modelo nas escalas quantitativa (itens 21, 16, 14 e 13) e qualitativa (itens 9, 21, 23 e 22). Foram localizadas pessoas com padrão errático de respostas, e cinco indivíduos tiveram pontuação mínima. Constatou-se discrepância entre dificuldade dos itens e habilidade da amostra, indicando que as habilidades dos indivíduos estavam abaixo da dificuldade dos itens. Houve correlação significativa entre a força de preensão do MSMA e as medidas de habilidade dos indivíduos na escala quantitativa $(\mathrm{r}=0,51 ; \mathrm{P}<0,0001)$ e qualitativa $(\mathrm{r}=0,57 ; \mathrm{P}<0,0001)$, e ainda entre as duas escalas nas medidas de habilidades dos individuos $(\mathrm{r}=0,97 ; \mathrm{P}<0,0001)$.

Conclusões. O MAL-Brasil apresenta potencial para avaliação do uso do MSMA de hemiplégicos crônicos brasileiros. Entretanto, apresenta limitação para uso em indivíduos com comprometimento severo do MSMA. Ainda, a validade de construto foi comprometida pela presença de itens erráticos. O MAL-Brasil deve ser aplicado em outras amostras para que sua validade seja mais amplamente investigada.

Palavras-chave Acidente cerebral vascular; psicometria; membro superior; validade dos testes; reprodutibilidade dos testes.

1 Universidade Federal de Minas Gerais (UFMG) Programa de Pós-Graduação em Ciências da Reabilitação, Belo Horizonte (MG), Brasil.

2 UFMG, Departamento de Terapia Ocupacional, Belo Horizonte (MG), Brasil.
UFMG, Departamento de Fisioterapia, Belo Horizonte (MG), Brasil. Correspondência: Luci Fuscaldi Teixeira-Salmela, lfts@ufmg.br

4 Universidade Federal de Pernambuco (UFPE), Departamento de Fisioterapia, Recife (PE), Brasil.
O acidente vascular encefálico (AVE) é uma das principais causas de incapacidade em adultos em idade economicamente ativa (1-3), sendo apontado como 
um dos maiores problemas de saúde na América Latina (4). Um estudo com foco na sobrecarga das doenças identificou o AVE como a terceira causa líder de anos de vida ajustados por incapacidade (disability-adjusted life years, DALY) — ou seja, a soma dos anos de vida perdidos por mortalidade prematura e dos anos vividos com incapacidade ajustada pela severidade - em países desenvolvidos, e a sexta causa em todo o mundo (5). No Brasil, enquanto a tendência de mortalidade por AVE apresentou declínio em três das cinco regiões brasileiras (6), é significativa a contribuição dessa doença para os DALY (7).

O comprometimento do uso do membro superior mais afetado (MSMA) nas tarefas manuais de vida diária é uma queixa frequente de indivíduos após o AVE; após 6 meses, de 25,0 a 53,0\% permanecem dependentes em pelo menos uma tarefa que envolve o uso unilateral ou bilateral dos membros superiores (8, 9). Hedmam et al. (10) relataram que $67,0 \%$ dos indivíduos consideraram o não uso do MSMA como um dos maiores problemas. Todavia, a funcionalidade do MSMA tem sido avaliada pela observação do desempenho em testes executados, geralmente, em laboratórios ou clínicas $(8,11)$, o que não corresponde ao funcionamento do indivíduo no seu cotidiano.

Atualmente, existe um instrumento padronizado para avaliar a função e o uso espontâneo do MSMA: o Motor Activity $\log$ (MAL) (8, 11-14). O MAL leva em conta o "aprendizado do não uso" (15-17) do MSMA pelo paciente e a requisição funcional do MSMA nas atividades de vida diária. Estudos que investigaram as propriedades psicométricas desse instrumento reportaram estabilidade $(15,16)$, boa consistência interna e confiabilidade teste-reteste e validade adequadas (15-17). A versão original possui 14 itens (MAL-14) $(15,17)$. Contudo, para avaliação de indivíduos com maior comprometimento motor do MSMA (indivíduos com menor amplitude de movimento ativo e passivo do ombro, cotovelo, punho, dedos e polegar), foi desenvolvida a versão com 30 itens (MAL-30), que também aborda atividades manuais de vida diária $(12,16)$.

O MAL foi desenvolvido na língua inglesa; portanto, para ser utilizado no Brasil, são necessárias a sua tradução, adaptação transcultural e avaliação das propriedades psicométricas no contexto brasileiro $(12,18)$. Assim, os objetivos deste estudo foram: realizar a tradução do MAL para o português brasileiro e adaptar o MAL-30 para a cultura brasileira; examinar as propriedades psicométricas da escala em indivíduos brasileiros acometidos por AVE crônico (referidos neste estudo como hemiplégicos); identificar possíveis limitações do instrumento e, se necessário, recomendar adaptações ou a exclusão de itens que não apresentem propriedades psicométricas adequadas.

\section{MATERIAIS E MÉTODOS}

Este estudo metodológico foi realizado em duas etapas: 1) tradução do MAL para o português brasileiro, retrotradução, análise da versão traduzida pelo comitê de juízes especialistas e préteste da versão final; e 2) análise das propriedades psicométricas.

\section{$\mathrm{O}$ instrumento}

O MAL é um instrumento específico para avaliação da função do MSMA de hemiplégicos, aplicado através de entrevista $(11,13,19)$. Tanto o MAL-14 como o MAL-30 apresentam duas escalas ordinais, cada uma com seis pontos, para a graduação das atividades: uma escala se relaciona à quantidade e a outra à qualidade do uso do MSMA (12, 15-17). Na escala quantitativa, a pontuação varia de zero (não usa o MSMA) a cinco (usa o MSMA da mesma forma que usava antes do AVE) (12). Na escala qualitativa, a pontuação varia de zero (o MSMA não é usado de forma alguma para a atividade) a cinco (sua habilidade de usar o MSMA é tão boa quanto era antes do AVE) (12), com a possibilidade de pontuações intermediárias, como 0,5 ou 1,5, se esse detalhamento refletir melhor a realidade do uso do MSMA. A pontuação total é o cálculo da média para cada escala. Quanto maior a média, melhor a quantidade e a qualidade de uso do MSMA. Quando alguma atividade não se aplica ao indivíduo, descarta-se o item, e o cálculo da média é refeito (12).

Os indivíduos são esclarecidos sobre a finalidade do instrumento e a diferença entre as escalas; a seguir, avaliam a quantidade e a qualidade de uso do MSMA no seu contexto de vida $(11,12)$. Se alguma atividade não é realizada, é possível analisar e classificar o motivo através do registro de codificação $(11,12,19)$.

\section{Tradução e adaptação transcultural do MAL}

Após autorização dos autores que desenvolveram o instrumento e seguindo as recomendações de Beaton et al. (18), o MAL foi traduzido para o português brasileiro do ponto de vista semântico, cultural e conceitual, de forma independente, por duas tradutoras bilíngues, falantes nativas de português brasileiro. A síntese das versões traduzidas foi retrotraduzida de forma independente por dois outros tradutores falantes nativos de inglês. A versão final foi submetida a um comitê de especialistas composto por uma fisioterapeuta, professora universitária com domínio do tema do estudo e fluente nos dois idiomas, por dois professores de inglês, alfabetizados na língua inglesa e fluentes na língua portuguesa, e pelas duas tradutoras do instrumento. Esse comitê discutiu a clareza, a pertinência e a equivalência entre as versões traduzidas, retrotraduzidas e originais. Durante esse processo, nenhum item do instrumento foi modificado, pois todos foram considerados comuns no dia a dia da população brasileira.

A versão final, Motor Activity LogBrasil (MAL-Brasil), foi submetida a préteste em 10 hemiplégicos da comunidade. Nesse grupo havia cinco mulheres. A média de idade foi de 52,9 $\pm 13,0$ anos, e a média de tempo de AVE foi de 73,0 \pm 46,1 meses. O grupo não apresentou déficits cognitivos e afasia sensitiva, conforme o Mini-Exame do Estado Mental (MEEM) (ponto de corte de 18/19 para indivíduos analfabetos e de 24/25 para indivíduos com instrução escolar) (20). Nenhum problema relacionado a redação, clareza dos itens ou ambiguidade nas respostas foi encontrado. O MALBrasil e os códigos para pontuação das escalas estão apresentados no anexo 1.

\section{Participantes}

Para investigação das propriedades psicométricas foram recrutados 77 hemiplégicos da comunidade na região metropolitana de Belo Horizonte, Estado de Minas Gerais, Brasil. Foram feitos 153 contatos, por telefone ou pessoalmente, através de listas obtidas de profissionais da reabilitação e de projetos de pesquisa da Universidade Federal de Minas Gerais (UFMG). Dos 153 indivíduos contatados, 97 relataram dificuldade em usar o MSMA e concordaram em participar 
do estudo, sendo que 77 atenderam aos critérios de inclusão.

Os critérios de inclusão foram: AVE primário ou recorrente (há no mínimo 6 meses), idade superior a 20 anos e hemiplegia ou hemiparesia caracterizada pela presença de espasticidade dos músculos flexores de cotovelo (escala modificada de Ashworth) (21) ou pela fraqueza de preensão manual superior a 10,0\% (dinamômetro Jamar, ${ }^{\circledR}$ Enterprises Inc., Irvington, Nova Iorque, EUA) (22). Foram excluídos indivíduos com déficits cognitivos e afasia sensitiva, avaliados pelo MEEM (20), apraxia e negligência unilateral importante, examinadas pela execução de gestos simples (23), e AVE bilateral.

A força de preensão manual (média de três medidas em kgf) foi avaliada seguindo protocolos padronizados (24). A exterocepção foi avaliada com um chumaço de algodão, sendo questionado se a sensação percebida no MSMA era a mesma ou diferente do lado menos afetado (25). A propriocepção foi testada posicionando-se o polegar em locais diferentes e pedindo ao indivíduo para reconhecer sua posição sem auxílio da visão (25). Todos os participantes assinaram o termo de consentimento livre e esclarecido aprovado pelo Comitê de Ética em Pesquisa da UFMG (processo ETIC 564/07).

\section{Aplicação do MAL-Brasil}

Dados sociodemográficos e clínicos foram coletados dos 77 participantes, e o MAL-Brasil foi aplicado por um único examinador, devidamente treinado (14). Os indivíduos foram informados sobre o estudo e questionados sobre o uso do MSMA na semana anterior. Os indivíduos que relataram não ter usado o MSMA esclareceram o motivo, que foi adequadamente codificado. As avaliações foram realizadas na UFMG, em centros de reabilitação ou no domicílio dos participantes.

Dos 77 hemiplégicos analisados, 10 participaram da avaliação de confiabilidade teste-reteste. Esse grupo foi testado duas vezes, com intervalo de 7 dias entre as aplicações. Para investigação das demais propriedades psicométricas, como consistência interna, estabilidade, reprodutibilidade e validade de construto, foi utilizada a análise de Rasch. O cálculo amostral baseou-se na recomendação de 10 indivíduos para cada opção de escore do instrumento $(26,27)$. Assim, seriam necessários pelo menos 60 indivíduos para avaliação do MAL-Brasil.

\section{Análise estatística}

As análises estatísticas descritivas e os testes de normalidade foram realizados utilizando-se o programa Statistical Package for the Social Sciences (SPSS), versão 13.0. Testes $\mathrm{t}$ de Student para amostras independentes ou pareadas foram utilizados para comparar a força de preensão, considerando-se a dominância do MSMA e a diferença de força entre o MSMA e o membro menos afetado do mesmo indivíduo. A relação entre a idade e a medida de habilidade dos indivíduos na escala quantitativa foi examinada pela correlação de Pearson. A correlação de Spearman foi utilizada para avaliar as seguintes associações: correlação entre idade e medida da habilidade dos indivíduos na escala qualitativa; correlação entre tempo de AVE e força de preensão do MSMA e medidas de habilidade dos indivíduos em ambas as escalas; e a relação entre as escalas. O nível de significância foi de $\alpha<0,05$. A confiabilidade teste-reteste foi investigada pelo coeficiente de correlação intraclasse (CCI), sendo classificada segundo Portey e Watkins (28) como excelente $(>0,75)$, moderada $(0,50$ a 0,75$)$ ou baixa $(<0,50)$.

\section{Análise de Rasch}

A análise de Rasch, realizada com o programa Winsteps versão 3.68.2, calibra a dificuldade dos itens e o nível de habilidade dos indivíduos em um mesmo contínuo linear simples, dividido em intervalos iguais (logits) $(26,29)$. Quanto maior a quantidade e a qualidade de uso do MSMA, maior a probabilidade de o indivíduo atingir escores altos em todos os itens (fáceis ou difíceis). Quanto mais fácil o item, maior a probabilidade de qualquer pessoa atingir um escore alto $(26,29)$. Isso significa que o teste se enquadra no modelo de medida $(26,29)$. Esses princípios se aplicam se o conjunto de itens estiver medindo uma habilidade unidimensional (26).

A unidimensionalidade do instrumento foi aferida pelos valores de mean square residuais (MnSq) e pelo valor $z$, que expressam a relação entre o escore esperado e o obtido $(26,29)$. Os valores razoáveis, que indicam adequação dos itens do instrumento, são $\mathrm{MnSq}=1 \pm 0,3$, com valor de $z= \pm 2$. Um valor de $\mathrm{MnSq}$ alto indica escores variáveis ou erráticos (nesse caso, pessoas com pior habilidade receberiam escores altos nos itens difíceis ou vice-versa) $(26,29)$. Isso sugere que o item não combina com os outros para medir um contínuo de habilidade, ou que existem problemas na sua definição (26, 29). Um valor de $\mathrm{MnSq}<0,7 \operatorname{com} z<-2$ indica redundância ou pouca variabilidade de escores (26). O primeiro resultado (MnSq alto) representa ameaça para a validade, enquanto que o segundo (MnSq $<0,7 \operatorname{com} z<-2$ ) indica que o item não discrimina pessoas com diferentes níveis funcionais $(26,29)$. Nesse sentido, foram revisados os itens com $\mathrm{MnSq}>1,3$ e valor associado de $z \geq 2$ em dois formatos, infit ou outfit, que sinalizam flutuações nas pontuações e a presença de escores extremos, respectivamente (26). Uma situação na qual mais que 5,0\% do número total de itens não se enquadram no modelo indica que o conjunto de itens não serve para medir um conceito unidimensional (26). Esse mesmo raciocínio é usado para examinar a qualidade das medidas obtidas para cada indivíduo entrevistado $(26,29)$.

O modelo de Rasch fornece ainda o valor do erro associado à ordenação de cada item e às medidas dos indivíduos, estimando um "índice de separação", ou seja, permite determinar em quantos níveis de habilidade os itens separam a amostra $(26,30)$. Para adequar-se à expectativa do modelo de Rasch, espera-se que um teste possa dividir os participantes em pelo menos dois níveis de habilidade, e os itens em pelo menos dois níveis de dificuldade.

Finalmente, o teste de Rasch avalia o índice de confiabilidade dos indivíduos e dos itens. Esse índice de confiabilidade indica se o padrão de respostas observado para cada indivíduo e se o padrão de escores observado para o conjunto dos itens pode ser replicado em amostras similares. Análogo ao alfa de Cronbach, o índice de confiabilidade apresenta valores de 0 a 1 , sendo os valores acima de 0,80 considerados aceitáveis (26).

\section{RESULTADOS}

A tabela 1 apresenta as características sociodemográficas e clínicas da amostra. Foram entrevistados 77 hemiplégicos, dos quais $41(53,2 \%)$ eram homens. A média de idade foi de $57,5 \pm 12,4$ anos.

Dos 77 entrevistados, $44(57,1 \%)$ eram casados ou viviam com um companheiro; $60(77,9 \%)$ não completaram o 
TABELA 1. Caracterização clínica dos hemiplégicos entrevistados para validação do Motor Activity Log - Brasil, 2009

\begin{tabular}{|c|c|}
\hline Variável [No. (\%)] ${ }^{a}$ & Característica \\
\hline Idade (anos) & $57,5 \pm 12,4(21$ a 87$)$ \\
\hline \multicolumn{2}{|l|}{ Sexo } \\
\hline Masculino & $41(53,2)$ \\
\hline Feminino & $36(46,8)$ \\
\hline \multicolumn{2}{|l|}{ Tempo de AVE (meses) } \\
\hline AVE1b & $74,6 \pm 57,4$ (6 a 252) \\
\hline $\operatorname{AVE}^{c}(n=6)$ & $41,7 \pm 30,7(6$ a 84$)$ \\
\hline \multicolumn{2}{|l|}{ Tipo de AVE } \\
\hline Hemorrágico & $23(29,9)$ \\
\hline Isquêmico & $54(70,1)$ \\
\hline \multicolumn{2}{|l|}{ Membro superior dominante } \\
\hline Direito & $73(94,8)$ \\
\hline Esquerdo & $4(5,2)$ \\
\hline \multicolumn{2}{|l|}{ Membro superior mais afetado dominante } \\
\hline Não & $38(49,4)$ \\
\hline $\operatorname{Sim}$ & $39(50,6)$ \\
\hline Escore do Mini-Exame do Estado Mental & $26,2 \pm 3,7$ (18 a 30$)$ \\
\hline \multicolumn{2}{|l|}{ Dor no ombro } \\
\hline Ausente & $36(46,8)$ \\
\hline Repouso & $1(1,3)$ \\
\hline Movimento & $23(29,9)$ \\
\hline Repouso + movimento & $17(22,1)$ \\
\hline \multicolumn{2}{|l|}{ Exterocepção } \\
\hline Normal & $42(54,5)$ \\
\hline Diminuída & $34(44,2)$ \\
\hline Ausente & $1(1,3)$ \\
\hline \multicolumn{2}{|l|}{ Propriocepção } \\
\hline Normal & $60(77,9)$ \\
\hline Diminuída & $09(11,7)$ \\
\hline Ausente & $8(10,4)$ \\
\hline \multicolumn{2}{|l|}{ Tônus muscular } \\
\hline 0 & $15(19,5)$ \\
\hline 1 & $15(19,5)$ \\
\hline $1+$ & $16(20,8)$ \\
\hline 2 & $22(28,6)$ \\
\hline 3 & $09(11,7)$ \\
\hline
\end{tabular}

ensino fundamental e sete $(9,1 \%)$ tinham curso superior; $20(26,0 \%)$ relataram depressão; $39(50,6 \%)$ realizavam tratamento fisioterapêutico; $30(39,0 \%)$ eram aposentados; e três $(4,0 \%)$ mantinham-se em atividade profissional.

Houve diferença estatisticamente significativa $(\mathrm{t}=-15,18 ; P<0,0001)$ entre as medidas de força de preensão do MSMA $(10,2 \pm 8,7 \mathrm{kgf})$ e do membro superior menos afetado $(29,0 \pm 9,2 \mathrm{kgf})$. Não houve diferença estatisticamente significativa $(P=0,54)$ entre a força de preensão dos indivíduos com o MSMA dominante $(9,3 \pm 7,3 \mathrm{kgf})$ e não dominante $(11,0 \pm 10,0 \mathrm{kgf})$.

\section{Confiabilidade teste-reteste}

O CCI foi de 0,98 para as escalas qualitativa e quantitativa do MAL-Brasil considerando-se as pontuações totais. Os itens individuais obtiveram CCI variando de 0,44 a 1,00 na escala quantita- tiva ( $>0,80$ em $90,0 \%$ dos itens) e de 0,15 a 1,00 na escala qualitativa $(>0,80 \mathrm{em}$ $87,0 \%$ dos itens).

\section{Análise de Rasch}

A calibração dos itens, de mais fáceis a mais difíceis, para as duas escalas é apresentada nas tabelas 2 e 3 . Na escala quantitativa, o item 23 foi o mais difícil $(1,08$ logits) e o 10 foi o mais fácil $(-2,14$ logits $)$ (tabela 2). Na escala qualitativa, o item 9 foi o mais difícil (1,01 logits) e o 12 foi o mais fácil $(-1,36$ logits) (tabela 3$)$.

Os valores médios de MnSq infit e outfit na escala quantitativa $(1,07, z=0,1 \mathrm{e}$ $0,86, z=-0,3$, respectivamente) e na escala qualitativa $(1,12, z=0,1$ e 0,87 , $z=-0,3$, respectivamente) sinalizam que o conjunto dos itens enquadrou-se no modelo. A análise individual indicou que quatro itens $(13,3 \%)$ não se encaixaram nas expectativas do modelo nas escalas quantitativa (itens $21,16,14$ e 13) e quali- tativa (itens 9, 21, 23 e 22). Alguns itens foram previsíveis nas duas escalas (tabelas 2 e 3). Foram localizadas pessoas com padrão errático de respostas, e cinco indivíduos tiveram pontuação mínima.

No geral, as atividades mais fáceis estão calibradas na região inferior, e as mais difíceis na região superior (tabelas 2 e 3). Itens como "colocar e tirar meias" e "tirar o telefone do gancho" parecem posicionados de maneira inversa ao que seria esperado.

$\mathrm{Na}$ análise sem os indivíduos com escore minimo o índice de separação dos indivíduos e itens foi de 3,46 (cinco níveis de habilidade) e 4,76 (sete níveis de dificuldade) na escala quantitativa, e 3,39 (cinco níveis de habilidade) e 3,74 (cinco níveis de dificuldade) na escala qualitativa, respectivamente. $\mathrm{O}$ índice de confiabilidade foi de 0,92 para os indivíduos nas duas escalas, e de 0,96 e 0,93 na escala quantitativa e qualitativa, respectivamente, para os itens. Considerando-se os indivíduos com escore mínimo, o índice de separação das pessoas indicou três níveis de habilidade (2,34 na escala quantitativa e 2,29 na qualitativa), com confiabilidade de 0,85 e 0,84 nas escalas quantitativa e qualitativa, respectivamente.

A figura 1 representa os contínuos de quantidade e qualidade de uso à esquerda e de dificuldade dos itens à direita. No topo, aparecem itens à direita com poucos indivíduos alinhados. $\mathrm{Na}$ parte inferior, é possível observar indivíduos com escore zero, sem presença de itens alinhados à direita. As pessoas concentraram-se na região inferior (menor habilidade), e os itens, na região superior (maior dificuldade). Essa discrepância entre dificuldade dos itens e habilidade da amostra é evidenciada pela diferença entre a calibração média (M) dos itens (definida como zero) e a média dos indivíduos (MI) (-1,59 logits na escala quantitativa e $-1,72$ logits na qualitativa). As médias negativas das pessoas indicam que suas habilidades estavam abaixo da dificuldade dos itens. Os indivíduos estão diferenciados de acordo com sexo, idade e dominância do MSMA.

Houve correlação estatisticamente significativa entre a força de preensão do MSMA e as medidas de habilidade dos indivíduos na escala quantitativa $(r=$ $0,51 ; P<0,0001)$ e qualitativa $(r=0,57$; $P<0,0001)$. Também houve correlação significativa entre as duas escalas nas medidas de habilidades dos indivíduos $(r=0,97 ; P<0,0001)$. 
TABELA 2. Calibração dos 30 itens da escala quantitativa do Motor Activity Log - Brasil, 2009

\begin{tabular}{|c|c|c|c|c|c|c|}
\hline \multirow[b]{2}{*}{ Item } & \multirow[b]{2}{*}{ Calibraçãoc } & \multirow[b]{2}{*}{ Erro } & \multicolumn{2}{|c|}{ Infit $^{\mathrm{a}}$} & \multicolumn{2}{|c|}{ Outfit $^{\mathrm{b}}$} \\
\hline & & & $\mathrm{MnSq}^{\mathrm{d}}$ & $z^{e}$ & $\mathrm{MnSq}^{\mathrm{d}}$ & $z^{e}$ \\
\hline 23) Usar chave para destrancar porta & 1,08 & 0,20 & 1,33 & 0,9 & 0,44 & $-0,6$ \\
\hline 9) Utilizar controle remoto da TV & 1,08 & 0,20 & 1,47 & 1,2 & 1,63 & 0,9 \\
\hline 21) Escovar os dentes ${ }^{f}$ & 0,88 & 0,18 & 1,81 & 2,1 & 0,77 & $-0,1$ \\
\hline 4) Tirar o telefone do gancho & 0,86 & 0,18 & 1,13 & 0,5 & 0,45 & $-0,6$ \\
\hline 26) Usar garfo ou colher para alimentar-se & 0,53 & 0,15 & 1,29 & 1,1 & 0,64 & $-0,5$ \\
\hline 28) Levantar xícara pela alça & 0,53 & 0,15 & 0,88 & $-0,4$ & 0,54 & $-0,7$ \\
\hline 27) Pentear o cabelo & 0,52 & 0,15 & 1,12 & 0,5 & 0,63 & $-0,5$ \\
\hline 5) Passar pano na bancada da cozinha ou outra superfície & 0,37 & 0,13 & 0,85 & $-0,6$ & 0,9 & 0,1 \\
\hline 8) Abrir porta girando a maçaneta & 0,33 & 0,13 & 1,23 & 1,0 & 0,95 & 0,1 \\
\hline 16) Tirar os sapatos ${ }^{\dagger}$ & 0,33 & 0,13 & 1,51 & 2,0 & 1,04 & 0,3 \\
\hline 22) Colocar base de maquiagem, loção ou creme de barbear no rosto & 0,30 & 0,13 & 1,39 & 1,6 & 0,93 & 0,1 \\
\hline 11) Abrir/fechar torneira de rosca ou alavanca & 0,20 & 0,13 & 0,75 & $-1,2$ & 0,60 & $-0,8$ \\
\hline 14) Tirar as meias ${ }^{f}$ & 0,14 & 0,12 & 1,56 & 2,4 & 0,87 & $-0,1$ \\
\hline 20) Levantar copo, garrafa ou lata & 0,11 & 0,12 & 0,66 & $-1,8$ & 0,51 & $-1,1$ \\
\hline 6) Sair do carro & 0,06 & 0,12 & 1,09 & 0,5 & 0,95 & 0,1 \\
\hline 24) Escrever no papel & 0,03 & 0,17 & 1,24 & 0,9 & 1,19 & 0,5 \\
\hline 1) Acender a luz pelo interruptor & 0,02 & 0,12 & 0,78 & $-1,1$ & 0,93 & 0,0 \\
\hline 17) Levantar-se da cadeira com apoio de braço & 0,01 & 0,12 & 1,13 & 0,7 & 0,98 & 0,1 \\
\hline 2) Abrir gaveta & $-0,02$ & 0,12 & 0,80 & $-1,0$ & 0,94 & 0,0 \\
\hline 3) Tirar peça de roupa da gaveta & $-0,02$ & 0,12 & 0,84 & $-0,8$ & 0,94 & 0,0 \\
\hline 19) Puxar cadeira em direção à mesa após estar assentado & $-0,06$ & 0,12 & 1,01 & 0,1 & 0,70 & $-0,7$ \\
\hline 30) Comer metade sanduíche, tira-gosto ou petisco & $-0,06$ & 0,12 & 0,81 & $-1,0$ & 0,63 & $-0,9$ \\
\hline 7) Abrir geladeira & $-0,13$ & 0,11 & 1,02 & 0,2 & 1,18 & 0,6 \\
\hline 18) Afastar cadeira da mesa antes de sentar-se & $-0,24$ & 0,11 & 0,83 & $-0,9$ & 0,64 & $-1,0$ \\
\hline 15) Calçar os sapatos & $-0,60$ & 0,11 & 1,00 & 0,1 & 1,30 & 1,0 \\
\hline 13) Colocar as meias ${ }^{f}$ & $-0,62$ & 0,11 & 1,93 & 4,1 & 1,68 & 2,0 \\
\hline 29) Abotoar uma camisa & $-0,64$ & 0,11 & 1,18 & 1,0 & 0,90 & $-0,3$ \\
\hline 25) Carregar objeto na mão & $-0,83$ & 0,10 & 0,78 & $-1,3$ & 0,87 & $-0,4$ \\
\hline 12) Secar as mãos 9 & $-2,01$ & 0,11 & 0,46 & $-3,7$ & 0,62 & $-2,2$ \\
\hline 10) Lavar as mãos ${ }^{g}$ & $-2,14$ & 0,12 & 0,31 & $-5,2$ & 0,40 & $-4,0$ \\
\hline
\end{tabular}

a Grau de adequação ao modelo mais sensível a variações no escore, próximas do nível de habilidade das pessoas ou do nível de dificuldade do item.

b Grau de adequação do item ou indivíduo que é mais sensível a escores extremos.

c 1,08 = mais difícil; $-2,14$ = mais fácil.

d Resíduo transformado da diferença entre o escore esperado e o observado, que sinaliza o grau de distorção no sistema de medida. O valor esperado é 1 , sendo aceitáveis variações de $\pm 0,3$.

e Valores estandardizados da estatística de adequação dos itens com média $=0$ e variância $=1$. Valores dentro do limite $z= \pm 2$ são aceitáveis $(P<0,05)$.

f Itens erráticos com $\mathrm{MnSq}>1,3 ; z \geq 2$.

$\mathrm{g}$ Itens previsíveis com $\mathrm{MnSq}<0,7 ; z<-2$.

\section{DISCUSSÃO}

Neste estudo, o MAL foi traduzido e adaptado transculturalmente para o português brasileiro (18), e suas propriedades psicométricas foram investigadas. Durante a primeira etapa, não foi necessária a modificação dos itens e foi mantida a versão final avaliada pelo comitê de especialistas. Os resultados da segunda etapa evidenciaram que o MAL apresenta potencial para aplicação clínica na avaliação do MSMA de hemiplégicos crônicos brasileiros. Entretanto, o instrumento apresentou limitação para indivíduos com baixos níveis de função do MSMA, e sua validade foi comprometida pela presença de itens erráticos.

A confiabilidade teste-reteste para ambas as escalas apresentou valores excelentes nas pontuações totais e para a maioria dos itens (28). Os índices de confiabilidade da análise de Rasch, tanto para os itens como para os indivíduos, foram altos; isso significa que as respostas dos indivíduos podem ser reproduzidas em aplicações subsequentes do instrumento $(26,28)$. Os participantes foram divididos em cinco níveis de habilidades, enquanto os itens foram divididos em sete níveis de dificuldade na escala quantitativa e cinco na qualitativa. Isso demonstra que o instrumento cumpriu a expectativa de dividir os indivíduos em pelo menos dois níveis de habilidade, e os itens em dois níveis de dificuldade. $\mathrm{Na}$ análise que incluiu os indivíduos com escore mínimo, a estabilidade de calibração e o índice de separação foram mais baixos, devido ao erro de medida, que é maior quando se incluem os indivíduos para os quais a escala é muito difícil.

Comportamento errático foi verificado em quatro itens $(13,3 \%)$ para cada uma das escalas. Possíveis razões foram: uso de estratégias compensatórias (indivíduos apresentavam comprometimentos crônicos) $(31,32)$, auxílio da família (11,
32) e variabilidade de pontuação devido a características individuais (pessoas com dificuldade no item avaliaram o uso acima do esperado para sua habilidade ou vice-versa) $(29,32)$. No item "escovar os dentes", os indivíduos usavam o lado menos afetado devido à maior destreza $\mathrm{e}$ à presença de dor no ombro mais afetado $(26,29)$. No item "tirar os sapatos", muitos indivíduos usavam somente o membro superior menos afetado ou os pés, preferindo calçados mais fáceis de tirar. O item "colocar as meias" não foi uma atividade habitual, por ser o Brasil um país de clima mais quente (29). O item "utilizar o controle remoto da TV" foi pouco realizado com o MSMA, devido à pouca destreza manual, sendo preferido o uso do membro superior menos afetado. Apesar de os indivíduos não apresentarem déficits cognitivos, alguns demonstraram dificuldade em recordar as atividades realizadas $(29,32)$. O nível de escolaridade mais baixo também dificul- 
TABELA 3. Calibração dos 30 itens da escala qualitativa do Motor Activity Log - Brasil, 2009

\begin{tabular}{|c|c|c|c|c|c|c|}
\hline \multirow[b]{2}{*}{ Item } & \multirow[b]{2}{*}{ Calibraçãoc } & \multirow[b]{2}{*}{ Erro } & \multicolumn{2}{|c|}{ Infit $^{\mathrm{a}}$} & \multicolumn{2}{|c|}{ Outfit $^{\mathrm{b}}$} \\
\hline & & & $\mathrm{MnSq}^{\mathrm{d}}$ & $z^{e}$ & $\mathrm{MnSq}^{\mathrm{d}}$ & $z^{e}$ \\
\hline 9) Utilizar controle remoto da TV ${ }^{f}$ & 1,01 & 0,19 & 1,86 & 2,2 & 1,08 & 0,4 \\
\hline 21) Escovar os dentes ${ }^{f}$ & 0,93 & 0,18 & 2,04 & 2,7 & 1,14 & 0,5 \\
\hline 23) Usar chave para destrancar porta & 0,93 & 0,18 & 1,76 & 2,1 & 0,70 & 0,0 \\
\hline 26) Usar garfo ou colher para alimentar-se & 0,68 & 0,16 & 1,50 & 1,7 & 0,79 & 0,0 \\
\hline 4) Tirar o telefone do gancho & 0,63 & 0,16 & 1,35 & 1,3 & 0,71 & $-0,1$ \\
\hline 27) Pentear o cabelo & 0,56 & 0,15 & 1,21 & 0,9 & 0,85 & 0,0 \\
\hline 16) Tirar os sapatos & 0,48 & 0,14 & 1,13 & 0,6 & 1,02 & 0,3 \\
\hline 28) Levantar xícara pela alça & 0,48 & 0,14 & 1,11 & 0,5 & 0,74 & $-0,2$ \\
\hline 24) Escrever no papel & 0,35 & 0,19 & 0,98 & 0,0 & 1,04 & 0,4 \\
\hline 22) Colocar base de maquiagem, loção ou creme de barbear no rosto ${ }^{f}$ & 0,31 & 0,13 & 1,70 & 2,7 & 1,21 & 0,5 \\
\hline 14) Tirar as meias & 0,26 & 0,13 & 1,33 & 1,5 & 0,80 & $-0,2$ \\
\hline 5) Passar pano na bancada da cozinha ou outra superfície & 0,12 & 0,13 & 1,21 & 1,0 & 1,10 & 0,4 \\
\hline 8) Abrir porta girando a maçaneta & 0,09 & 0,12 & 1,34 & 1,6 & 1,28 & 0,7 \\
\hline 11) Abrir/fechar torneira de rosca ou alavanca & 0,08 & 0,12 & 0,95 & $-0,2$ & 0,88 & $-0,1$ \\
\hline 6) Sair do carro & 0,02 & 0,12 & 1,16 & 0,8 & 1,14 & 0,4 \\
\hline 20) Levantar copo, garrafa ou lata & $-0,10$ & 0,12 & 0,74 & $-1,4$ & 0,57 & $-1,1$ \\
\hline 30) Comer metade sanduíche, tira-gosto ou petisco & $-0,11$ & 0,12 & 0,87 & $-0,6$ & 0,69 & $-0,7$ \\
\hline 3) Tirar peça de roupa da gaveta & $-0,17$ & 0,12 & 1,19 & 1,0 & 1,05 & 0,3 \\
\hline 2) Abrir gaveta & $-0,18$ & 0,12 & 0,91 & $-0,4$ & 0,74 & $-0,6$ \\
\hline 19) Puxar cadeira em direção à mesa após estar assentado & $-0,18$ & 0,12 & 0,81 & $-1,0$ & 0,59 & $-1,1$ \\
\hline 1) Acender a luz pelo interruptor & $-0,25$ & 0,11 & 1,40 & 1,9 & 1,22 & 0,7 \\
\hline 7) Abrir geladeira & $-0,26$ & 0,11 & 1,14 & 0,8 & 1,04 & 0,2 \\
\hline 17) Levantar-se da cadeira com apoio de braço & $-0,28$ & 0,11 & 1,37 & 1,8 & 1,07 & 0,3 \\
\hline 29) Abotoar uma camisa & $-0,30$ & 0,11 & 0,78 & $-1,2$ & 0,66 & $-1,0$ \\
\hline 18) Afastar cadeira da mesa antes de sentar-se & $-0,37$ & 0,11 & 0,69 & $-1,8$ & 0,54 & $-1,5$ \\
\hline 13) Colocar as meias & $-0,44$ & 0,11 & 1,30 & 1,6 & 1,16 & 0,6 \\
\hline 15) Calçar os sapatos 9 & $-0,51$ & 0,11 & 0,59 & $-2,7$ & 0,56 & $-1,7$ \\
\hline 25) Carregar objeto na mãog & $-1,10$ & 0,10 & 0,68 & $-2,2$ & 0,78 & $-1,1$ \\
\hline 10) Lavar as mãosg & $-1,32$ & 0,11 & 0,29 & $-6,0$ & 0,45 & $-3,6$ \\
\hline 12) Secar as mãos ${ }^{g}$ & $-1,36$ & 0,11 & 0,37 & $-5,0$ & 0,55 & $-2,7$ \\
\hline
\end{tabular}

a Grau de adequação ao modelo mais sensível a variações no escore, próximas do nível de habilidade das pessoas ou do nível de dificuldade do item.

b Grau de adequação do item ou indivíduo que é mais sensível a escores extremos.

c $1,01=$ mais difícil; $-1,36=$ mais fácil.

d Resíduo transformado da diferença entre o escore esperado e o observado, que sinaliza o grau de distorção no sistema de medida. O valor esperado é 1 , sendo aceitáveis variações de $\pm 0,3$.

e Valores estandardizados da estatística de adequação dos itens com média $=0$ e variância $=1$. Valores dentro do limite $z= \pm 2$ são aceitáveis $(P<0,05)$.

${ }^{\dagger}$ Itens erráticos com $\mathrm{MnSq}>1,3 ; z \geq 2$.

$g$ Itens previsíveis com $\mathrm{MnSq}<0,7 ; z<-2$.

tou o entendimento das instruções do teste (29). Souza et al. (29) e Penta et al. (32) também discutiram algumas dessas interferências para explicar itens erráticos encontrados em seus estudos.

Quanto à ordem dos itens na escala, observamos que, nos itens 4 e 14, que são de execução mais fácil, muitos indivíduos preferiram usar somente o membro menos afetado, devido à maior destreza e velocidade para realizar essas atividades. No item 13, mais difícil, muitas pessoas usaram o MSMA para auxiliar o membro superior menos afetado. Os itens 13 e 14 apresentaram comportamento errático, indicando variabilidade nas respostas. A análise das pessoas também indicou que a pontuação está sujeita a variação de acordo com diferentes características individuais e situações, que devem ser observadas na interpretação dos resultados (29).

Os itens previsíveis, "secar e lavar as mãos", nas duas escalas, são atividades de vida diária comuns. Para essas ativi- dades, mesmo as pessoas com menor habilidade usaram o MSMA. Penta et al. (32) também apontaram, após análise de Rasch, que o item considerado mais fácil pelos hemiplégicos foi "lavar as mãos".

Verificamos que cinco indivíduos apresentaram pontuação mínima. No mapa da distribuição dos indivíduos e itens, houve concentração de indivíduos na parte inferior, sem presença de itens alinhados à direita. Isso indica que os itens não cobriram pessoas com menor habilidade. No topo, observaram-se itens difíceis para a amostra, sem efeito teto, indicando que a aplicação do instrumento é possível em indivíduos com maior habilidade $(26,29)$. Os participantes distribuíram-se pelo contínuo das escalas, não existindo padrão específico relacionado a idade, sexo e lado acometido. Harris e Eng (31) reportaram que hemiplégicos crônicos desenvolvem estratégias adaptativas que compensam a limitação do MSMA, independentemente do lado aco- metido. Neste estudo, a diferença de força de preensão foi estatisticamente significativa entre os membros superiores, mas não houve diferença significativa considerando a dominância do MSMA, como já relatado em estudos anteriores $(31,33)$.

Os indivíduos com grande limitação da função do MSMA, na maioria, apresentaram diferença percentual de força de preensão acima de 75,0\%. A importância da força de preensão foi verificada pela correlação estatisticamente significativa com as medidas de habilidades dos indivíduos nas escalas (8). Harris e Eng (8) apontaram que a força de preensão do MSMA e a força muscular total do membro apresentaram correlação estatisticamente significativa com os escores do MAL, sendo responsáveis por $78,0 \%$ da variância nos escores. Canning et al. (34) reportaram que fraqueza muscular e diminuição da destreza foram os fatores que mais contribuíram para a incapacidade funcional do MSMA após o AVE. 
FIGURA 1. Mapa representativo da distribuição dos indivíduos e itens no contínuo de níveis de quantidade $(A)$ e qualidade $(B)$ de uso do membro superior mais afetado conforme o Motor Activity Log - Brasil, 2009a

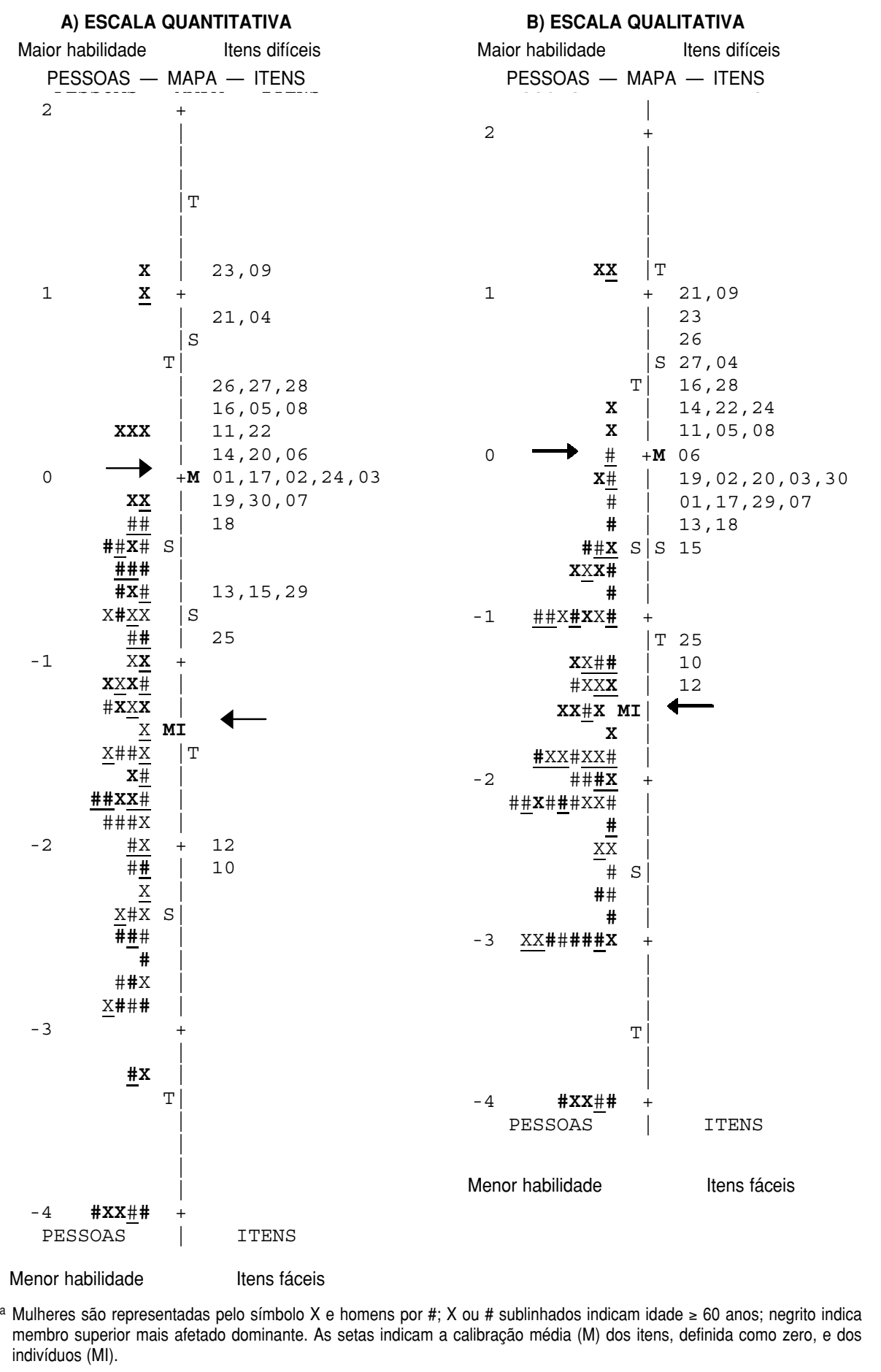

Neste estudo, muitos indivíduos evitaram o uso do MSMA devido à dor no ombro, apesar de apresentarem habilidade nas atividades. Chae et al. (35) não encontraram associação significativa entre dor no ombro e desempenho de atividades. Entretanto, ressaltaram que os instrumentos utilizados analisaram o dronizados, incentivos dos terapeutas e equipamentos adaptados). Isso expõe a importância da avaliação do MSMA no cotidiano dos hemiplégicos $(11,32)$.

Os resultados deste estudo refletem o uso do MSMA de hemiplégicos crônicos da comunidade. O MAL-Brasil deve ser aplicado em outras amostras, que incluam indivíduos com menor comprometimento do MSMA. Se o número de itens erráticos permanecer superior a 5,0\%, como encontrado no presente estudo, pode haver a necessidade de modificações do instrumento (adaptação/exclusão de itens), com nova investigação das propriedades psicométricas. Quanto à aplicação clínica, o MAL-Brasil apresenta potencial para avaliação do uso do MSMA por hemiplégicos crônicos em conjunto com outros testes padronizados. Entretanto, apresenta limitações para indivíduos com grande comprometimento do MSMA, pois não apresentou itens fáceis o suficiente para avaliar esses indivíduos mais comprometidos. Sugere-se que o entrevistador reforce as instruções iniciais e fique atento para a interpretação dos resultados e a análise das respostas dos itens erráticos.

Uma das limitações deste estudo foi o fato de não ter sido aplicado um instrumento para avaliar e classificar a habilidade motora dos indivíduos incluídos na análise de Rasch, especialmente diante da constatação de que o instrumento demonstrou limitação para indivíduos com grande diferença percentual de força de preensão manual. Além disso, a confiabilidade interavaliadores não foi avaliada.

Concluindo, o MAL-Brasil apresentou evidência de excelente confiabilidade teste-reteste e estabilidade na calibração dos itens e indivíduos e permitiu a discriminação de indivíduos em diferentes níveis de uso do MSMA. Entretanto, apresentou limitação quando utilizado em indivíduos com grande comprometimento do MSMA, e sua validade de construto foi comprometida por itens erráticos. Portanto, o MAL-Brasil deve ser aplicado em outras amostras para que sua validade seja mais amplamente investigada.

Agradecimentos. Às agências de fomento nacionais Coordenação de Aperfeiçoamento de Pessoal de Nível Superior (CAPES), Conselho Nacional de Desenvolvimento Científico e Tecnológico $(\mathrm{CNPq})$ e Fundação de Amparo à Pesquisa do Estado de Minas Gerais (FAPEMIG), pelo apoio financeiro. 


\section{REFERÊNCIAS}

1. Cieza A, Ewert T, Ustun T, Chatterii S, Kostanjsek N, Stucki G. Development of ICF Core Sets for patients with chronic conditions. J Rehabil Med. 2004;36(44):9-11.

2. Doyle PJ. Measuring health outcomes in stroke survivors. Arch Phys Med Rehabil. 2002;83(12):39-43.

3. Lessa I. Epidemiologia das doenças cerebrovasculares no Brasil. Rev Soc Cardiol Estado de São Paulo. 1999;9(4):509-18.

4. Lavados PM, Hennis AJM, Fernandes JG, Medina MT, Legetic B, Hoppe A, et al. Stroke epidemiology, prevention, and management strategies at a regional level: Latin America and the Caribbean. Lancet Neurol. 2007;6(4): 362-72.

5. Murray CJ, Lopez AD. Global mortality, disability, and the contribution of risk factors: global burden of disease study. Lancet. 1997; 349(9063):1436-42.

6. Souza MFM, Alencar AP, Malta DC, Moura L, Mansur AP. Análise de séries temporais da mortalidade por doenças isquêmicas do coração e cerebrovasculares, nas cinco regiões do Brasil, no período de 1981 a 2001. Arq Bras Cardiol. 2006;87(6):135-40.

7. Mackay J, Mensah GA. The atlas of heart disease and stroke. Genebra: World Health Organization; 2004.

8. Harris JE, Eng JJ. Paretic upper-limb strength best explains arm activity in people with stroke. Phys Ther. 2007;87(1):88-97.

9. Pang MY, Harris JE, Eng JJ. A communitybased upper-extremity group exercise program improves motor function and performance of functional activities in chronic stroke: a randomized controlled trial. Arch Phys Med Rehabil. 2006;87(1):1-9.

10. Hedman LD, Sullivan JE, Hilliard MJ, Brown DM. Neuromuscular electrical stimulation during task-oriented exercise improves arm function for an individual with proximal arm dysfunction after stroke. Am J Phys Med Rehabil. 2007;86(7):592-6.

11. Uswatte G, Taub E. Implications of the learned nonuse formulations for measuring rehabilitation outcomes: lessons from constraint-induced movement therapy. Rehabil Psychol. 2005;50(1):34-42.

12. Saliba VA, Chaves Júnior IP, Faria CDCM, Teixeira-Salmela LF. Propriedades psicométricas da Motor Activity Log: uma revisão sistemática da literatura. Fisioter Mov. 2008;21(3):59-67.

13. Taub E, Uswatte G, Pidikiti R. ConstraintInduced Movement Therapy: a new family of techniques with broad application to physical rehabilitation-a clinical review. J Rehabil Res Dev. 1999;36(3):237-51.

14. Taub E, Uswatte G, Morris D. Upper Extremity Motor Activity Log [manual]. Available from Edward Taub, Psychology Department, UAB, Birmingham, AL, EUA; 2004.

15. Uswatte G, Taub E, Morris D, Vignolo M, McCulloch K. Reliability and validity of the upper-extremity Motor Activity Log-14 for measuring real-world arm use. Stroke. 2005; 36(11):2493-6.

16. Uswatte G, Taub E, Morris D, Light K, Thompson PA. The Motor Activity Log-28: assessing daily use of the hemiparetic arm after stroke. Neurology. 2006;67(7):1189-94

17. Van Der Lee JH, Beckerman H, Knol DL, de Vet HC, Bouter LM. Clinimetric properties of the Motor Activity Log for the assessment of arm use in hemiparetic patients. Stroke. 2004;35(6):1410-4.

18. Beaton DE, Bombardier C, Guillermin F, Ferraz MB. Guidelines for the process of cross-cultural adaptation of self-report measures. Spine. 2000;25(24):3186-91.

19. Taub E, Miller NE, Novack TA, CooK EW Fleming WC, Nepomuceno CS, et al. Technique to improve chronic motor deficit after stroke. Arch Phys Med Rehabil. 1993;74(4): 347-54.

20. Lourenco RA, Veras RP. Mini-Exame do Estado Mental: características psicométricas em idosos ambulatoriais. Rev Saude Publica. 2006;40(4):712-9.

21. Brashear A, Zafonte R, Corcoran M, GalvezJimenez N, Gracies JM, Gordon MF, et al. Inter- and intrarater reliability of the Ashworth Scale and the Disability Assessment Scale in patients with upper-limb poststroke spasticity. Arch Phys Med Rehabil. 2002; 83(10):1349-54

22. Incel NA, Ceceli E, Durukan PB, Erdem HR, Yorgancioglu ZR. Grip strength: effect of hand dominance. Singapore Med J. 2002;43 (5):234-7.

23. Azouvi P, Bartolomeo P, Beis JM, Perennou D, Pradat-Diehl P, Rosseeaux M. A battery of tests for the quantitative assessment of unilateral neglect. Restor Neurol Neurosci. 2006;24 (4-6):273-85.

24. Figueiredo IM, Sampaio RF, Mancini MC, Silva FCM, Souza MAP. Teste de força de preensão utilizando o dinamômetro Jamar. Acta Fisiatrica. 2007;14(2):104-10.

25. Sunderland A, Tinson D, Bradley L, Hewer RL. Arm function after stroke. An evaluation of grip strength as a measure of recovery and a prognostic indicator. J Neurol Neurosurg Psychiatry. 1989;52(11):1267-72.

26. Bond TG, Fox CM. Applying the Rasch Model: fundamental measurement in the human sciences. Mahwah, NJ: Lawrence Erlbaum Associates; 2001.

27. Wallen M, Bundy A, Pont K, Ziviani J. Psychometric properties of the Pediatric Motor Activity Log used for children with cerebral palsy. Dev Med Child Neurol. 2008;51(17): 200-8.

28. Portney LG, Watkins MP. Foundations of clinical research: application to practice. $2^{\mathrm{a}}$ ed. Upper Saddle River, NJ: Prentice-Hall; 2000

29. Souza AC, Magalhães LC, Teixeira-Salmela LF. Adaptação transcultural e análise das propriedades psicométricas da versão brasileira do Perfil de Atividade Humana. Cad Saude Publica. 2006;22(12):2623-36.

30. Wright BD, Master GN. Rating scale analysis: Rasch measurement. Chicago: MESA; 1982.

31. Harris JE, Eng JJ. Individuals with the dominant hand affected following stroke demonstrate less impairment than those with the nondominant hand affected. Neurorehabil Neural Repair. 2006;20(3):380-9.

32. Penta M, Tesio L, Arnould C, Zancan A, Thonnard JL. The ABILHAND Questionnaire as a measure of manual ability in chronic stroke patients: Rasch-based validation and relationship to upper limb impairment. Stroke. 2001;32:1627-34.

33. Mercier C, Bourbonnais D. Relative shoulder flexor and handgrip strength is related to upper limb function after stroke. Clin Rehabil. 2004;18(2):215-21.

34. Canning CG, Ada L, Adams R, O'Dwyer NJ. Loss of strength contributes more to physical disability after stroke than loss of dexterity. Clin Rehabil. 2004;18(3):300-8.

35. Chae J, Mascarenhas D, Yu DT, Kirsteins A, Elovic EP, Flanagan SR, et al. Poststroke shoulder pain: its relationship to motor impairment, activity limitation, and quality of life. Arch Phys Med Rehabil. 2007;88:298-301.

Manuscrito recebido em 12 de março de 2011. Aceito em versão revisada em 4 de abril de 2011. 
ANEXO 1. Versão Final da Motor Activity Log - Brasil

\begin{tabular}{|c|c|c|c|}
\hline Nome & Data & & \\
\hline \multirow[t]{2}{*}{ Examinador } & Lado dominante & Lado acometido & \\
\hline & & $\begin{array}{c}\text { Escala } \\
\text { Quantitativa }\end{array}$ & $\begin{array}{c}\text { Escala } \\
\text { Qualitativa }\end{array}$ \\
\hline
\end{tabular}

2) Abrir uma gaveta

3) Tirar uma peça de roupa da gaveta

4) Tirar o telefone do gancho

5) Passar um pano (limpar) na bancada da cozinha ou outra superfície

6) Sair do carro (inclui apenas o movimento necessário para levantar do banco e ficar em pé fora do carro, depois que a porta estiver aberta)

7) Abrir a geladeira

\section{8) Abrir uma porta girando a maçaneta}

9) Utilizar o controle remoto da TV

10) Lavar as mãos (inclui ensaboar e enxaguar as mãos; não inclui abrir/fechar uma torneira manual)

11) Abrir e fechar uma torneira de rosca ou alavanca

12) Secar as mãos

13) Colocar as meias

14) Tirar as meias

15) Calçar os sapatos (inclui amarrar os cadarços e ajustar os velcros ou as tiras)

16) Tirar os sapatos (inclui desamarrar cadarços e soltar os velcros ou as tiras)

17) Levantar-se de uma cadeira com apoio de braço

18) Afastar a cadeira da mesa antes de se assentar

19) Puxar a cadeira em direção à mesa após estar assentado

20) Levantar um copo, garrafa (de vidro ou plástico) ou lata (não precisa incluir beber)

21) Escovar os dentes (não inclui a preparação da escova de dente ou escovar a dentadura, a menos que esta seja escovada dentro da boca)

22) Colocar base de maquiagem, loção ou creme de barbear no rosto

23) Usar uma chave para destrancar uma porta

24) Escrever no papel (se a mão utilizada para escrever antes do derrame é a mais afetada, pontue o item; se a mão que não escrevia antes do derrame é a mais afetada, pule o item e assinale $\mathrm{N} / \mathrm{A}$ )

25) Carregar um objeto na mão (dependurar um item sobre o braço não é aceitável)

26) Usar um garfo ou uma colher para se alimentar (se refere à ação de levar a comida até a boca com o garfo ou colher)

27) Pentear o cabelo

\section{8) Levantar uma xícara pela alça}

29) Abotoar uma camisa

30) Comer a metade de um sanduíche, tira-gosto ou petiscos (qualquer alimento que se come com a mão)
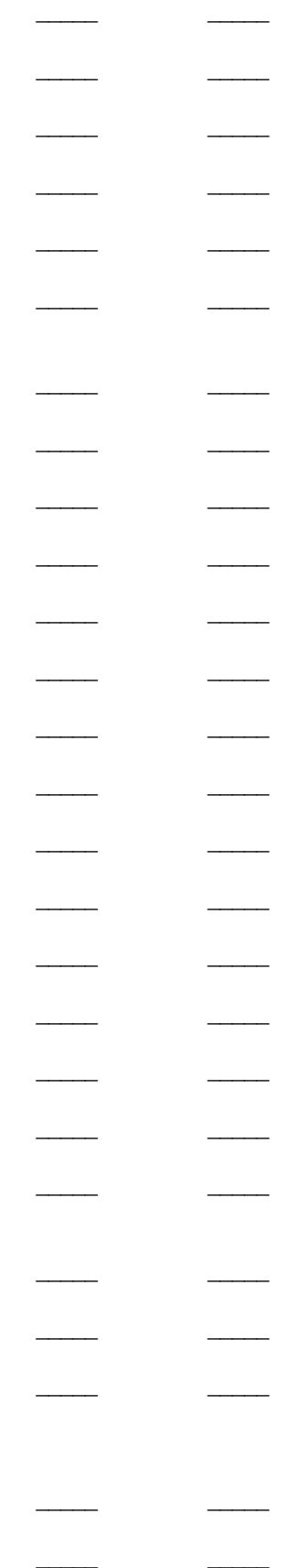

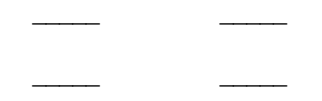

TOTAL:

Se não, por quê? (use código)_ Comentários

Se não, por quê? (use código) Comentários

Se não, por quê? (use código)_ Comentários

Se não, por quê? (use código)_

Comentários

Se não, por quê? (use código) Comentários

Se não, por quê? (use código) Comentários

Se não, por quê? (use código)_ Comentários

Se não, por quê? (use código) Comentários

Se não, por quê? (use código) Comentários

Se não, por quê? (use código) Comentários

Se não, por quê? (use código)_ Comentários

Se não, por quê? (use código)_ Comentários

Se não, por quê? (use código)_ Comentários

Se não, por quê? (use código)_ Comentários

Se não, por quê? (use código)_ Comentários

Se não, por quê? (use código_

Comentários

Se não, por quê? (use código) Comentários

Se não, por quê? (use código)_ Comentários

Se não, por quê? (use código) Comentários

Se não, por quê? (use código)_ Comentários

Se não, por quê? (use código)_ Comentários

Se não, por quê? (use código)_ Comentários

Se não, por quê? (use código) Comentários

Se não, por quê? (use código)_ Comentários

Se não, por quê? (use código)_ Comentários

Se não, por quê? (use código)_ Comentários

Se não, por quê? (use código)_ Comentários

Se não, por quê? (use código) Comentários

Se não, por quê? (use código) Comentários

Se não, por quê? (use código) Comentários 
ANEXO 1. (Continuação)

Códigos para registro de respostas "não"

1. "Eu usei o braço não afetado o tempo todo" (assinale "0").

2. "Outra pessoa fez por mim". (assinale "0").

3. "Eu nunca faço esta atividade, com ou sem ajuda de outros porque é impossível." Por exemplo, pentear o cabelo em pessoas carecas. (assinale "N/A" e retire da lista de itens).

4. "Eu às vezes faço esta atividade, mas não tive a oportunidade desde a última vez em que eu respondi essas perguntas." (repita o último valor assinalado para esta atividade).

5. Hemiparesia de mão não-dominante. (somente aplicável para a questão 24; assinale "N/A" e retire da lista de itens).

\begin{tabular}{|c|c|}
\hline ESCALA QUANTITATIVA & ESCALA QUALITATIVA \\
\hline 0 - Não usei o braço mais fraco (não usei) & $\begin{array}{l}0 \text { - O braço mais fraco não foi usado de forma alguma para } \\
\text { aquela atividade (nunca) }\end{array}$ \\
\hline .5 & .5 \\
\hline $\begin{array}{l}\text { 1- Ocasionalmente usei o braço mais fraco, apenas muito rara- } \\
\text { mente (muito raramente). }\end{array}$ & $\begin{array}{l}1 \text { - O braço mais fraco se moveu durante aquela atividade, } \underline{\text { mas }} \\
\text { não ajudou (muito fraco). }\end{array}$ \\
\hline 1.5 & 1.5 \\
\hline $\begin{array}{l}2 \text { - } \dot{A} \text { s vezes usei o braço mais fraco, mas fiz a atividade a maior } \\
\text { parte do tempo com meu braço mais forte (raramente) }\end{array}$ & $\begin{array}{l}2 \text { - O braço mais fraco foi de alguma utilidade durante esta ativi- } \\
\text { dade, porém, precisou de ajuda do braço mais forte ou se moveu } \\
\text { muito lentamente ou com dificuldade (fraco). }\end{array}$ \\
\hline 2.5 & 2.5 \\
\hline $\begin{array}{l}\text { 3- Usei o braço mais fraco aproximadamente metade das vezes } \\
\text { que usava antes do derrame (metade pré-derrame). }\end{array}$ & $\begin{array}{l}\text { 3- O braço mais fraco foi utilizado com o propósito indicado, } \\
\text { porém os movimentos foram lentos ou foram efetuados apenas } \\
\text { com algum esforço (bom) }\end{array}$ \\
\hline 3.5 & 3.5 \\
\hline $\begin{array}{l}4 \text { - Usei o braço mais fraco quase o mesmo tanto que antes do } \\
\text { derrame (3/4 pré-derrame) }\end{array}$ & $\begin{array}{l}4 \text { - Os movimentos feitos pelo braço mais fraco foram quase nor- } \\
\text { mais, mas não chegaram a ser tão rápidos ou precisos quanto o } \\
\text { normal (quase normal) }\end{array}$ \\
\hline 4.5 & 4.5 \\
\hline $\begin{array}{l}\text { 5- Usei o braço mais fraco com a mesma freqüência de antes do } \\
\text { derrame (mesmo que pré-derrame) }\end{array}$ & $\begin{array}{l}\text { 5- A habilidade de usar o braço mais fraco para esta atividade foi } \\
\text { tão bom quanto antes do derrame (normal) }\end{array}$ \\
\hline
\end{tabular}

ABSTRACT Objectives. To describe the translation into Brazilian Portuguese and cross-cultural adaptation of the Motor Activity Log (MAL), an instrument specifically designed to assess function of the more severely affected upper limb in hemiplegics.

\section{Cross-cultural adaptation and analysis of the psychometric properties of the Brazilian version of the Motor Activity Log}

Methods. The MAL was translated and adapted according to standardized procedures and submitted to test-retest reliability assessment (intraclass correlation coefficient, ICC). Other psychometric properties were investigated using Rasch analysis in 77 chronic hemiplegics ( 41 men, mean age $=57.5 \pm 12.4$ years).

Results. An excellent ICC (0.98) was obtained for the total scores of both the quantity and quality MAL scales. When assessing degree of difficulty, "using a key to unlock the door" was the most difficult item on the quantity scale, whereas "washing hands" was the easiest one. On the quality scale, the most difficult item was "using the TV remote control," and the easiest one was "drying hands." The analyses showed that the set of items as a whole fit into the model; however, the individual analyses indicated that four items did not meet the expectations of the model in both the quantity (items 21, 16, 14, and 13) and quality (items 9, 21, 23, and 22) scales. Irregular response patterns were observed, and five subjects obtained the minimum score. There was disagreement between item difficulty and sample ability, suggesting that the abilities of individuals were below the degree of difficulty of the assessed items. A significant correlation was observed between grip strength of the more severely affected upper limb and motor skill measurement among individuals on the quantity $(r=0.51, P<0.0001)$ and quality scales $(r=0.57, P<0.0001)$, and also between the two scales when measuring the individuals' motor skills $(r=0.97$, $P<0.0001)$.

Conclusions. MAL-Brazil is potentially useful to evaluate the more severely affected upper limb in Brazilian patients with chronic hemiplegia. However, the instrument has limitations for use with individuals with severe limb impairments. Also, construct validity was affected by the presence of irregular score patterns. MAL-Brazil should be applied to additional samples to further investigate its validity.

Key words Stroke; psychometrics; upper extremity; validity of tests; reproducibility of results. 